\title{
Eficiência Bionutricional de Animais da Raça Nelore e seus Mestiços com Caracu, Angus e Simental
}

\section{Kepler Euclides Filho1, Geraldo Ramos de Figueiredo², Valéria Pacheco Batista Euclides ${ }^{3}$, Luiz Otávio Campos da Silva4, Viviane Queiroz Cusinato ${ }^{5}$}

\begin{abstract}
RESUMO - Foram avaliados 23 machos inteiros de três grupos genéticos, sete da raça Nelore (N), oito 1/2 Caracu + 1/4 Angus $+1 / 4$ Nelore $(\mathrm{CCAN})$ e oito $1 / 2 \mathrm{Caracu}+1 / 4$ Simental +1/4 Nelore (CCSN). Esses animais pertenciam a um projeto em desenvolvimento na Embrapa Gado de Corte, com o objetivo de identificar um grupo genético que, além de ser adaptado às condições tropicais e subtropicais, produza carne de qualidade de forma eficiente e competitiva (Projeto composto Indo-Euro). O presente experimento teve como objetivo específico avaliar a eficiência bionutricional desses animais. Para tanto, as variáveis ganho de peso e consumo de matéria seca foram consideradas simultaneamente em uma análise bivariada. Utilizando o maior autovalor, estabeleceu-se a primeira função discriminante canônica que foi usada para a obtenção da eficiência bionutricional. A análise estatística revelou efeito significativo de grupo genético sobre essa eficiência. As médias foram comparadas por meio dos seguintes contrastes: $\mathrm{C}_{1}$ ) CCAN versus CCSN; e $\mathrm{C}_{2}$ ) média dos animais CCAN e CCSN versus N. Como os dois contrastes foram significativos, concluiu-se haver diferenças entre os desempenhos nutricionais dos animais mestiços, e que os animais CCANs apresentaram melhor EBN do que os CCSNs (60,72 versus 48,63, respectivamente). Os mestiços apresentaram melhor eficiência do que os nelores (54,67 versus 16,70). As médias de quadrados mínimos para consumo de matéria seca diário e para ganho de peso médio diário, durante o período de avaliação, foram, respectivamente: 7,62 kg e $1 \mathrm{~kg} ; 7,19 \mathrm{~kg}$ e $1,23 \mathrm{~kg}$ e $7,57 \mathrm{~kg}$ e $1,15 \mathrm{~kg}$ para os animais $\mathrm{N}, \mathrm{CCAN}$ e CCSN.
\end{abstract}

Palavras-chave: bovino de corte, consumo, cruzamentos, eficiência bionutricional, ganho de peso

\section{Evaluation of Nellore and Nellore Crosses with Caracu, Angus and Simmental}

ABSTRACT - The data utilized in this study were obtained from 23 intacted animals from three different genetic groups: seven Nellore (N), eight $1 / 2$ Caracu $+1 / 4$ Angus $+1 / 4$ Nellore (CCAN) and eight $1 / 2$ Caracu $+1 / 4$ Simental $+1 / 4$ Nellore (CCSN). These animals were produced as part of an ample project which is being carried out at Embrapa Gado de Corte whose main objective is to identify a genetic group that besides being adapted, is capable to produce meat of high quality in an efficient and competitive way (Indo-Euro composite project). This particular experiment had the objective of evaluating the bio-nutritional efficiency (EBN) of such animals. In order to do that, the variables weight gain and dry matter consumption were jointly analyzed under a bivariate model. Using the higher eigenvalue, it was established the first discriminant canonical function, which, in turn, was utilized for obtaining the EBN values. The statistical analysis revealed to exist a significant effect of genetic group on EBN. The EBN averages were compared by two different contrasts: $\mathrm{C}_{1}$ ) CCAN versus $\mathrm{CCSN}$; and $\mathrm{C}_{2}$ ) average for CCAN and CCSN versus $\mathrm{N}$. Since both contrasts were significant it was concluded that there is difference between the performance of the two crossbred groups and, that the CCAN animals presented better EBN than the CCSN ones (60.72 versus 48.63, respectively). The F1's animals, on the other side, presented better bio-nutritional efficiency than Nellore (54.67 versus 16,70$)$. The least square means for daily dry matter consumption during the evaluation period as well as for average daily gain were, respectively, 6.47 $\mathrm{kg}$ and $1.00 \mathrm{~kg}, 7.90 \mathrm{~kg}$ and $1.23 \mathrm{~kg}$, and $7.57 \mathrm{~kg}$ and $1.15 \mathrm{~kg}$ for N, CCAN and CCSN animals, respectively.

Key Words: beef cattle, bio-nutritional efficiency, crossbreeding, feed consumption, weight gain

\section{Introdução}

A integração entre os diversos segmentos da cadeia produtiva da carne bovina, associada ao aumento da exigência do consumidor quanto à qualidade do produto final, tem demandado animais que sejam capa- zes de produzir carne macia de forma bioeconomicamente viável. Entre as alternativas disponíveis para atender a essas demandas, ressaltam-se os cruzamentos, que, segundo Euclides Filho (1997), se apresentam como importante opção para a melhoria da produção e da produtividade do setor de pecuária de corte.

\footnotetext{
${ }^{1}$ Eng.-Agr., Ph.D., CREA No 12.153/D, Visto 1.466/MS, Embrapa Gado de Corte, BR 262 km 4, Caixa Postal 154, CEP 79002-970 Campo Grande MS. Correio eletrônico: kepler@cnpgc.embrapa.br

${ }^{2}$ Eng.-Agr., M.Sc., CREA No 11.753/D-Visto 1.527/MS, Embrapa Gado de Corte. Correio eletrônico: geraldo@cnpgc.embrapa.br

${ }^{3}$ Enga.-Agra., Ph.D., CREA No 12.797/D, Embrapa Gado de Corte. Correio eletrônico: val@cnpgc.embrapa.br

4 Zoot., Ph.D., CRMV-MS No 0022-Z, Embrapa Gado de Corte. Correio eletrônico: locs@cnpgc.embrapa.br

${ }^{5}$ Bolsista da Fundação de Apoio à Pesquisa Agropecuária e Ambiental (Fundapam), Caixa Postal 154, CEP $79002-970$ Campo Grande, MS.
} 
Todavia, tal expectativa só será alcançada quando a combinação de raças for capaz de resultar em uma composição que atenda à prioridade final do negócio, isto é, a rentabilidade. Para isso, é necessário que os animais resultantes do cruzamento produzam carne de qualidade a custos que viabilizem sua competitividade tanto no mercado interno quanto no externo. Nesse contexto, os cruzamentos, envolvendo alguma raça européia adaptada, podem trazer grande contribuição se combinados com alguma outra raça européia não-adaptada, como forma de melhorar não só o desempenho, mas também a carcaça, em particular, sua conformação.

O cruzamento entre animais de raças européias não-adaptadas e zebuínas, e mesmo entre as européias adaptadas e zebuínas, possibilita a obtenção de indivíduos que, além de apresentarem bom desempenho ponderal, produzem carne macia (Razzok et al., 1986; Nardon et al., 1996, Euclides Filho et al. 1997; Perotto et al., 1998). Assim, a produção de indivíduos com composição racial que possibilite a combinação de $75 \%$ de genótipo europeu com $75 \%$ de genótipo adaptado (europeu adaptado x $1 / 2$ europeu não-adaptado $+1 / 2$ zebu) ou $75 \%$ e $50 \%$, respectivamente (europeu não-adaptado x $1 / 2$ europeu adaptado $+1 / 2$ zebu) pode ser capaz de atender a essas expectativas e de contribuir para o estabelecimento de sistemas de produção sustentáveis e competitivos.

Nesse sentido, este trabalho teve como objetivo avaliar a eficiência bionutricional, característica fundamental para a produção de carne, em animais mestiços com $75 \%$ de genótipo europeu e com $75 \%$ de genótipo adaptado. Esse índice tem se mostrado eficiente para avaliações de desempenho nutricional como indicam alguns resultados (Guidoni, 1994; Euclides Filho et al., 2001).

\section{Material e Métodos}

Foram utilizados 23 machos inteiros, de três grupos genéticos, sendo sete da raça Nelore $(\mathrm{N})$, oito $1 / 2$ Caracu $+1 / 4$ Angus $+1 / 4$ Nelore (CCAN) e oito $1 / 2$ Caracu $+1 / 4$ Simental $+1 / 4$ Nelore (CCSN). Esses animais pertenciam a um projeto em desenvolvimento na Embrapa Gado de Corte, com o objetivo de identificar um grupo genético adaptado que produza carne de qualidade de forma eficiente e competitiva (Projeto composto Indo-Euro).

Desde a gestação até a desmama, realizada entre seis e sete meses de idade, todos os animais foram mantidos em pastagens de Brachiaria decumbens, recebendo como suplementação alimentar apenas sal mineral, fornecido à vontade. Após a desmama, todos os bezerros permaneceram por mais 70 dias no mesmo tipo de pastagem; no entanto, receberam uma suplementação alimentar à base de concentrados $(0,8 \%$ do peso vivo $)$ e ajustada a cada 28 dias, de acordo com os pesos vivos obtidos mensalmente. Essa ração era constituída por uma mistura de 73,48\% de milho triturado, $24,49 \%$ de farelo de soja, $1,96 \%$ de uréia pecuária e $0,07 \%$ de rumensin, com base na matéria seca (MS).

Após esse período, os animais foram alojados em uma baia coletiva por quinze dias, com o objetivo de melhorar o manejo e adaptá-los à alimentação. Posteriormente, foram distribuídos, de forma aleatória, em baias individuais providas de bebedouro automático e cochos para fornecimento de sal mineral e ração. Durante os 152 dias de período experimental, além dos 15 dias em baia coletiva, os animais receberam uma ração constituída por silagem de milho e concentrado. O concentrado era composto de $41 \%$ de milho triturado, $56 \%$ de farelo de soja, $2,98 \%$ de calcário calcítico e $0,02 \%$ de rumensin e fornecido de modo a representar $30 \%$ da matéria seca ofertada.

A quantidade da ração era ajustada a cada três dias, para que sobrassem cerca de $10 \%$, como forma de garantir consumo ad libitum. A ração total era distribuída em dois períodos, e, todos os dias, tanto o fornecido como a sobra eram pesados. Para acompanhamento do desempenho e melhor ajuste da ração, os animais eram pesados mensalmente.

As variáveis ganho de peso e consumo de alimento têm correlação entre si e, por isso, foram consideradas de forma conjunta, em uma análise bivariada, utilizando o procedimento "MANOVA" existente no SAS (1989). Segundo Guidoni (1994), esse procedimento resulta em um método mais adequado para avaliação do desempenho nutricional do que a conversão e a eficiência alimentar. Para complementar a análise, usou-se a primeira função discriminante canônica. As comparações entre os valores obtidos foram realizadas por meio dos seguintes contrastes: $\mathrm{C}_{1}$ ) CCAN versus CCSN; e $\mathrm{C}_{2}$ ) média de CCAN e CCSN versus $\mathrm{N}$.

Os valores dessa função foram definidos como eficiência bionutricional (Euclides Filho et al., 2001). Segundo esses mesmos autores, a utilização desse método pode ser capaz de detectar eventuais nãolinearidades da correlação existente entre consumo e ganho de peso nos diferentes grupos genéticos. 


\section{Resultados e Discussão}

A análise bivariada, envolvendo o ganho de peso e o consumo de matéria seca, revelou diferenças significativas $(\mathrm{P}<0,01)$ para as eficiências bionutricionais observadas nos diferentes grupos genéticos (Tabela 1). As médias de quadrados mínimos para a eficiência bionutricional encontram-se na Tabela 2 .

Os dois contrastes analisados, $\mathrm{C}_{1}$ e $\mathrm{C}_{2}$, apresentaram efeitos significativos $(\mathrm{P}<0,01)$. Dessa forma, observou-se que os animais CCANs apresentaram melhor eficiência bionutricional do que os CCSNs $(60,72$ versus 48,63$)$. No entanto, ambos os mestiços foram superiores aos nelores $(54,67$ versus 16,70$)$.

$\mathrm{Na}$ ausência de restrições, segundo Frisch \& Vercoe $(1977,1978)$, as diferenças de desempenho entre raças são freqüentemente observadas e estão, quase sempre, associadas ao consumo de alimentos, ao metabolismo basal e ao requerimento para mantença dos animais.

Nesse contexto, a superioridade dos animais CCANs parece contraditória, especialmente, no que se refere ao desempenho dos animais CCSNs que ocuparam a posição intermediária. Esse grupo genético, pela contribuição da raça Simental, teoricamente, deveria apresentar maior tamanho adulto e maior ganho de peso. Todavia, o que se observou (Tabela 3 ) foi que os animais CCANs apresentaram, além da melhor eficiência bionutricional, melhores ganhos diários médios de peso no período $(\mathrm{P}<0,05)$ do que os CCSNs $(1,25$ quilo/dia versus 1,15 quilo/dia, respectivamente). Analisando-se os consumos voluntários (Tabela 3 ), verificou-se que esses não foram diferentes para os dois grupos de mestiços (7,90 quilos de MS/dia versus 7,57 quilos de MS/dia para CCAN e CCSN, respectivamente).

Tabela 1 - Resumo da análise de variância da eficiência bionutricional

Table 1 - Summary of the analyses of variance for bio-nutritional efficiency

\begin{tabular}{lcc}
\hline $\begin{array}{l}\text { Fonte de variação } \\
\text { Source of variation }\end{array}$ & GL & $\begin{array}{c}\text { Quadrado médio } \\
\text { Mean square }\end{array}$ \\
\hline $\begin{array}{l}\text { Grupo genético } \\
\text { Genetic group }\end{array}$ & 2 & $147,95^{1}$ \\
$\begin{array}{l}\text { Idade inicial } \\
\text { Initial age }\end{array}$ & 1 & 185,65 \\
$\begin{array}{l}\text { Peso inicial } \\
\text { Initial weight }\end{array}$ & 1 & $2.394,77^{1}$ \\
$\begin{array}{l}\text { Resíduo } \\
\text { Error }\end{array}$ & 18 & 86,16 \\
${ }_{1}^{1} \mathrm{P}<0,05(P<.05)$. & &
\end{tabular}

R. Bras. Zootec., v.31, n.1, p.331-334, 2002 (suplemento)
Esse resultado, no entanto, está de acordo com vários outros apresentados em uma revisão publicada pelo NRC (1996). É possível observar nessa revisão que os animais da raça Simental apresentam requerimento de mantença superior àqueles verificados para animais da raça Angus. Dessa forma, parte maior da energia ingerida, no caso dos CCSNs, teria sido utilizada para atendimento de suas exigências para mantença do que aquela necessária para mantença dos CCANs e, por isso, estes apresentaram melhores ganhos de peso, evidenciando, claramente, o maior requerimento para mantença apresentado pelos animais CCSNs.

Euclides Filho et al. (2001), apesar de terem observado melhor eficiência bionutricional para animais F1s Angus-Nelore do que para animais F1s Simental-Nelore, não verificaram diferenças nos ganhos de peso médios diários, ou no consumo de MS médio diário. No entanto, a ração utilizada naquele trabalho era de qualidade inferior ao caso presente o que pode ter sido responsável, pelo menos em parte, por uma limitação de consumo dos animais "meiosangue" Simental-Nelore. Além disso, os animais avaliados, no experimento em pauta, são de grupos genéticos com maior porcentagem de contribuição de raças européias. Isso pode ter contribuído para o maior consumo e, conseqüentemente, maior ganho de peso.

Todavia, diferenças no desempenho nutricional de diferentes grupos genéticos nem sempre são observadas. Diversos autores, avaliando a conversão alimentar de grupos genéticos distintos, têm encontrado resultados que confirmam a inexistência de diferenças entre eles (Gonçalves et al., 1991; Euclides Filho et al., 1997). Entretanto, tais divergências são, segundo Vercoe (1967), bastante comuns em estudos de avaliações de eficiência alimentar envolvendo zebuínos e taurinos.

Tabela 2 - Médias de quadrados mínimos para eficiência bionutricional, conforme o grupo genético

Table 2 - Least square means for bio-nutritional effiency, according to the genetic group

\begin{tabular}{|c|c|}
\hline $\begin{array}{l}\text { rupo genético } \\
\text { enetic group }\end{array}$ & Bio-nutritional efficiency \\
\hline elore & 16,7 \\
\hline $\begin{array}{l}\text { Cellore } \\
\text { Caracu }+1 / 4 \text { Angus }+1 / 4 \text { Nelore }\end{array}$ & $60,72^{2}$ \\
\hline $\begin{array}{l}1 / 2 \text { Caracu }+1 / 4 \text { Angus }+1 / 4 \text { Nellore } \\
1 / 2 \text { Caracu }+1 / 4 \text { Simental }+1 / 4 \text { Nelore } \\
1 / 2 \text { Caracu }+1 / 4 \text { Simmental }+1 / 4 \text { Nellore }\end{array}$ & $48,63^{3}$ \\
\hline \multicolumn{2}{|c|}{$\begin{array}{l}\text { 1, 2, } 3 \text { Médias com sobrescritos diferentes diferem }(P<0,05) \text {, de } \\
\text { acordo com os contrastes analisados. } \\
\text { 1,2, } \begin{array}{l}\text { Means with different superscripts are different }(P<.05) \text {, according to the } \\
\text { analyzed contrasts. }\end{array}\end{array}$} \\
\hline
\end{tabular}


